\title{
NEW COUNTING FORMULA FOR DOMINATING SETS IN PATH AND CYCLE GRAPHS
}

\author{
Leomarich F. Casinillo \\ Department of Mathematics and Physics, Visayas State University, Visca, Baybay City, Leyte, Philippines \\ Email: leomarich_casinillo@yahoo.com
}

\begin{abstract}
Let $G=(V(G), E(G))$ be a path or cycle graph. A subset $D$ of $V(G)$ is a dominating set of $G$ if for every $u \in(V(G) \backslash D)$, there exists $v \in D$ such that $u v \in E(G)$, that is, $N[D]=V(G)$. The domination number of $G$, denoted by $\gamma(G)$, is the smallest cardinality of a dominating set of $G$. A set $D_{1} \subseteq V(G)$ is a set containing dominating vertices of degree 2, that is, each vertex is internally stable. A set $D_{2} \subseteq V(G)$ is a set containing dominating vertices where one of the element say $a \in D_{2}, \operatorname{deg}(a)=1$ and the rest are of degree 2. A set $D_{3} \subseteq V(G)$ is a set containing dominating vertices in which two of the elements say $b, c \in D_{3}, \operatorname{deg}(b)=\operatorname{deg}(c)=1$. This paper developed a new combinatorial formula that determines the number of ways of putting a dominating set in a path and cycle graphs of order $n \geq 1$ and $n \geq 3$, respectively. Further, a combinatorial function $P_{G}^{1}(n), P_{G}^{2}(n)$, and $P_{G}^{3}(n)$ that determines the probability of getting the set $D_{1}, D_{2}$, and $D_{3}$, respectively in graph $G$ of order $n$ were constructed.
\end{abstract}

Keywords: Dominating set, path, cycle, probability

\section{INTRODUCTION}

A connected graph $G$ is a pair of set $V(G)$ and $E(G)$, where $V(G)$ is a finite non-empty set called the vertex-set of $G$ and $E(G)$ is a set of unordered pair $\{a, b\}$ or simply $a b$ of distinct elements from $E(G)$ called the edge-set of $G$ where $a$ and $b$ are distinct elements in $V(G)$. Let $v \in V(G)$. The degree of vertex $v \in V(G)$, denoted by deg $(v)$ is the number of edges incident with $v$ in $G$. Then neighborhood of $v$ is the set $N_{G}(v)=N(v)=\{u \in V(G): u v \in E(G)\}$. If $X \subseteq V(G)$, then the open neighborhood of $X$ is the set $N_{G}(X)=N(X)=\cup_{v \in X} N_{G}(v)$. The closed neighborhood of $X$ is $N_{G}[X]=N[X]=X \cup N(X)$. A walk is a sequence $u_{1}, u_{2}, \ldots, u_{n}$ of vertices of graph $G$ such that $\left\{u_{i}, u_{i+1}\right\} \in E(G)$ for each $i=1,2, \ldots, n-1$. Vertices $u_{1}$ and $u_{n}$ are the endpoints of the walk while the vertices $u_{2}, u_{3}, \ldots, u_{n-1}$ are internal vertices of the walk. The length of walk is the number of edges on the walk, i.e., the walk $u_{1}, u_{2}, \ldots, u_{n}$ has length $n-1$. A path is a walk that does not repeat edges and does not end where it starts, i.e., $u_{1} \rightarrow u_{2} \rightarrow \ldots \rightarrow u_{n}, u_{1} \neq u_{n}$. A path of order $n$ and length $n-1$ is denoted by $P_{n}$ where $n \geq$ 1. A cycle is a walk that does not repeat edges and does end where it starts, i.e., $u_{1} \rightarrow u_{2} \rightarrow$ $\ldots \rightarrow u_{n} \rightarrow u_{1}$. A cycle graph of order $n$ and length $n$ is denoted by $C_{n}$ where $n \geq 3$. For other types of graphs, readers may refer to $[6,10]$. Let $a$ and $b$ be two distinct vertices in graph $G$. The distance $d_{G}(a, b)$ between two vertices $a$ and $b$ of a graph $G$ is defined as the length of the shortest walk between $a$ and $b$ in $G$. If there is no walk between $a$ and $b$ then we declare $d_{G}(a, b)=\infty$. The eccentricity $e(a)$ is the distance to a vertex farthest from $b$, that is, $e(a)=$ $\max \left\{d_{G}(a, b)\right\}$. For more information on the concepts of graphs, please refer to $[5,6,10]$. 
A subset $D$ of $V(G)$ is a dominating set of $G$ if for every $a \in V(G) \backslash D$, there exists $b \in D$ such that $a b \in E(G)$, i.e., $N[D]=V(G)$ where the elements of $D$ is called dominating vertices. The domination number of $G$, denoted by $\gamma(G)$, is the smallest cardinality of a dominating set of $G$. Let $I \subseteq G$. Then, $I$ is an independent dominating set if it is a dominating set in $G$ and no two vertices in set $I$ are adjacent. The independent domination number of $G$, denoted by $i(G)=$ $\gamma_{i}(G)$, is the smallest cardinality of an independent dominating set of $G$. The concepts of domination in graphs is investigated in $[1,4,7,9,11,12]$. In the study of Casinillo [3], the sum of two paths $P_{n}$ and $P_{m}$ is defined as connecting the last vertex of $P_{n}$ to the first vertex of $P_{m}$ and it is denoted as $P_{n} \Phi P_{n}=P_{n+m}$. Also, it is introduced that path of order $n$ with one dominating vertex at $i^{\text {th }}$ vertex is denoted by $P_{n}^{i}$. A set $D_{1} \subseteq V(G)$ is a set containing dominating vertices of degree 2 . A set $D_{2} \subseteq V(G)$ is a set containing dominating vertices where one of the element say $u \in D_{2}, \operatorname{deg}(u)=1$. A set $D_{3} \subseteq V(G)$ is a set containing dominating vertices in which two of the elements say $c, d \in D_{3}, \operatorname{deg}(c)=\operatorname{deg}(d)=1$. Hence, $D=$ $D_{1} \cup D_{2} \cup D_{3}$. Let $S_{G}$ be the set of all possible ways of getting dominating set $D$ in a graph $G$ and let $\varphi_{G}(n)=\left|S_{G}\right|$ be a function that determines the number of ways of putting dominating set in a graph $G$. In addition, let $f_{G}(n), g_{G}(n)$ and $h_{G}(n)$ be functions that determines the number of ways of putting set $D_{1}, D_{2}$ and $D_{3}$, respectively in a graph $G$, that is, $f_{G}(n)=f\left(D_{1}\right)$, $g_{G}(n)=g\left(D_{2}\right)$, and $h_{G}(n)=h\left(D_{3}\right)$. The probability of getting $D_{1}, D_{2}$ and $D_{3}$ in a graph $G$ are denoted by $P_{G}^{1}(n), P_{G}^{2}(n)$ and $P_{G}^{3}(n)$, respectively and defined as follows:

i.) $\quad P_{G}^{1}(n)=\operatorname{Prob}\left(D_{1}\right)=\frac{f_{G}(n)}{\varphi_{G}(n)}$;

ii.) $\quad P_{G}^{2}(n)=\operatorname{Prob}\left(D_{2}\right)=\frac{g_{G}(n)}{\varphi_{G}(n)}$; and

iii.) $\quad P_{G}^{3}(n)=\operatorname{Prob}\left(D_{3}\right)=\frac{h_{G}(n)}{\varphi_{G}(n)}$

where $P_{G}^{1}(n)+P_{G}^{2}(n)+P_{G}^{3}(n)=1$.

Currently, in the paper of Casinillo [3], the number of independent domination in path is determined using the Fibonacci binary string and in the paper of Samanmoo et al. [13], the $\gamma$ independent dominating graphs of paths and cycles were presented. Furthermore, Arocha and Llano [2] computed the number of dominating $k$-sets of paths, cycles and wheels using the concept of domination polynomials. Hence, the purpose of this paper is to present new counting formula for the number of dominating sets of path and cycle graphs that depends on the order of graph. In particular, a new combinatorial formula that determines the number of ways of putting a dominating set in a path and cycle graphs of order $n \geq 1$ and $n \geq 3$, respectively were developed. Furthermore, the combinatorial functions $P_{G}^{1}(n), P_{G}^{2}(n)$, and $P_{G}^{3}(n)$ that determines the probability of getting the set $D_{1}, D_{2}$, and $D_{3}$, respectively in graph $G$ were constructed.

\section{RESULTS}

We begin this section by considering a remark which is immediate from above definition of dominating vertex in graph $G$. This result determines the minimum domination number of path and cycle graphs.

Remark 2.1. [2,8] Let $P_{n}$ be a path of order $n \geq 1$ and $C_{n}$ be a cycle graph of order $n \geq 3$. Then, 


$$
\gamma\left(P_{n}\right)=\gamma\left(C_{n}\right)=\left\lceil\frac{n}{3}\right\rceil= \begin{cases}\frac{n}{3} & \text { if } n \equiv 0(\bmod 3) \\ \frac{n+2}{3} & \text { if } n \equiv 1(\bmod 3) \\ \frac{n+1}{3} & \text { if } n \equiv 2(\bmod 3)\end{cases}
$$

For path $P_{1}$ and $P_{2}$, it is clear that the dominating number is 1 . The next Theorem is a direct consequence of Remark 2.1. The Theorem is different meaning to the result of Casinillo [2] that deals with Fibonacci number of domination in path which only generates independent dominating sets. We have to note that in path and cycle graphs, $|D|=|I|$, however, the number of ways of getting set $D$ and $I$ are different. In particular, this Theorem determines the number of all possible ways of putting a dominating sets (connected and independent) in path graph $P_{n}$ which depends on the order $n$.

Theorem 2.1. Let $G=P_{n}$ where $n \in \mathbb{N}$. Then,

$$
\left|S_{G}\right|=\varphi_{G}(n)= \begin{cases}\frac{1}{18}\left(n^{2}+13 n+4\right) & \text { if } n \equiv 1(\bmod 3) \\ \frac{1}{3}(n+4) & \text { if } n \equiv 2(\bmod 3)\end{cases}
$$

Proof: To prove Theorem 2.1, we consider the following cases below:

Case 1. Let $G=P_{3 j}$ where $j \in \mathbb{N}$. By Remark 2.1, $\gamma(G)=j$ for all $j \in \mathbb{N}$ and implies that for all $u_{i} \in D, \bigcap_{\text {all } i} N\left(u_{i}\right)=\varnothing$ and $\operatorname{deg}\left(u_{i}\right)=2$. Thus, there is a unique configuration that for any $u_{j} \in D, \exists a, b \in V(G) \backslash D$ such that $N\left(u_{i}\right)=\{a, b\}$ and $d_{G}\left(u_{i}, u_{i+1}\right)=3$. Hence, it clearly follows that $\left|S_{G}\right|=\varphi_{G}(n=3 j)=1$.

Case 2. Let $G=P_{3 j+1}$ where $j \in\{0,1,2, \ldots\}$. Obviously, if $j=0$, then $n=1,|D|=|I|=1$ and $\left|S_{G}\right|=1$. By Remark 2.1, it follows that $\gamma(G)=j+1$ and we obtained the following subcases below:

Subcase 1. Firstly, there is a unique configuration of independent dominating set considering that $\exists u, v \in I=D$ such that $\operatorname{deg}(u)=\operatorname{deg}(v)=1$.

Subcase 2. Considering that there exists $a, b \in V(G) \backslash D$ and $\operatorname{deg}(a)=\operatorname{deg}(b)=1$ such that $N(a) \cup N(b) \subset D$. When $n=7$, there is 1 possibility that the arrangement of the independent dominating and non-dominating vertices are alternate. On the other hand, if $n \geq 10$, then there exists edge/s such that it is incident of two non-dominating vertices. Let $E_{i}$ be the edges. Note that $P_{2}^{2}$ and $P_{2}^{1}$ are in the first and last position of path $G$, respectively. Hence, $\left|V(G) \backslash V\left(P_{2}^{2} \Phi P_{2}^{1}\right)\right|=n-4$ and implies that the dominating number remaining is $\frac{n-4}{3}$. This follows that there are $\frac{n-7}{3}$ edges incident of two non-dominating vertices, that is, $E_{1}, E_{2}, \ldots, E_{\frac{n-7}{3}}$. Combinatorically speaking, the $\frac{n-4}{3}$ independent dominating vertices and edges $E_{1}, E_{2}, \ldots, E_{\frac{n-7}{3}}$ can be arranged in $\left(\frac{n-4}{3}\right)^{2}$ ways. However, the $\frac{n-4}{3}$ independent dominating vertices and edges $E_{1}, E_{2}, \ldots, E_{\frac{n-7}{3}}$ are not distinct, then the possible arrangement is given by

$$
\sum_{i=1}^{\frac{n-4}{3}} i=\frac{n^{2}-5 n+4}{18} .
$$


Subcase 3. Suppose that there exists $u \in D$ and $v \in V(G) \backslash D$ such that $\operatorname{deg}(u)=\operatorname{deg}(v)=1$ and $N(v) \subset D$, then there are $\frac{2(n-1)}{3}$ distinct arrangement can be form for $\gamma(G)=j+1$ by Remark 2.1.

Subcase 4. Lastly, if no vertex in $D$ is an end vertex in $G$, then $\exists u, v \in D$ such that $u v \in E(G)$. This implies that the position of vertex $u$ is in $P_{n}^{3 i-1}$ and vertex $v$ is in $P_{n}^{3 i}$ where $i \in$ $\left\{1,2, \ldots, \frac{n-1}{3}\right\}$. Clearly, there are $\frac{n-1}{3}$ configuration of this form.

Combining the 4 subcases, then it follows that

$$
\varphi_{G}(n=3 j+1)=1+\frac{n^{2}-5 n+4}{18}+\frac{2(n-1)}{3}+\frac{n-1}{3}=\frac{n^{2}+13 n+4}{18} .
$$

Case 3. Let $G=P_{3 j+2}$ where $j \in\{0,1,2, \ldots\}$. Clearly, if $n=2$, then there are 2 possible ways of generating a dominating set. Now we consider $n \geq 5$. By Remark 2.1, clearly, we have $\gamma(G)=j+1$, and again, we consider the following subcases below:

Subcase 1. If one of the vertex in $D$ has degree 1 , i.e., there exists $u \in V(G) \backslash D$ such that $\operatorname{deg}(u)=1$, then it follows that for all $u_{j+1} \in D, \bigcap_{\text {all } j} N\left(u_{j+1}\right)=\emptyset$. And it is concluded that there are 2 possible arrangement of this form in graph $G$.

Subcase 2. On the other hand, if for all $v_{j} \in D, \operatorname{deg}\left(v_{j}\right)=2$, then there exists edge/s $E_{i} \in E(G)$ such that it is incident with non-dominating vertices. Obviously, if $n=5$, then there is only 1 possible way to generate a dominating set in $G$. Now consider $n \geq 8$. Since $P_{2}^{2}$ and $P_{2}^{1}$ are in the first and last position of $G$, respectively, then $\left|V(G) \backslash V\left(P_{2}^{2} \Phi P_{2}^{1}\right)\right|=n-4$ and the dominating vertices remaining is $\frac{n-5}{3}$. So, there are $\frac{n-5}{3}$ edges incident of two non-dominating vertices, that is, $E_{1}, E_{2}, \ldots, E_{\frac{n-5}{3}}$. So, it follows that there are $\frac{n-5}{3}+1=\frac{n-2}{3}$ distinct configuration of dominating sets of this form in graph $G$. Combining the 2 subcases, then we end up with

$$
\varphi_{G}(n=3 j+2)=2+\frac{n-2}{3}=\frac{n+4}{3} .
$$

Hence, if we combine all three cases, then this completes the proof.

The following corollaries are immediate results of Theorem 2.1 above. The result shows the combinatorial formula of getting the number of ways of generating sets $D_{1}, D_{2}$, and $D_{3}$ in graph $G$. For a trivial graph $G=P_{1}$, sets $D_{1}, D_{2}$, and $D_{3}$ are not applicable.

Corollary 2.1. Let $G$ be a path of order $n \geq 3$. Then,

$$
f_{G}(n)=f\left(D_{1}\right)= \begin{cases}1 & \text { if } n \equiv 0(\bmod 3) \\ \frac{1}{18}\left(n^{2}+n-2\right) & \text { if } n \equiv 1(\bmod 3) \\ \frac{1}{3}(n-2) & \text { if } n \equiv 2(\bmod 3)\end{cases}
$$

Proof: Obvious from Theorem 2.1.

Corollary 2.2. Let $G$ be a path of order $n \geq 1$. Then,

$$
g_{G}(n)=g\left(D_{2}\right)= \begin{cases}0 & \text { if } n \equiv 0(\bmod 3) \\ \frac{2}{3}(n-1) & \text { if } n \equiv 1(\bmod 3) \\ 2 & \text { if } n \equiv 2(\bmod 3)\end{cases}
$$

Proof: Obvious from Theorem 2.1. 
Corollary 2.3. Let $G$ be a path of order $n \geq 4$. Then,

$$
h_{G}(n)=h\left(D_{3}\right)=\left\{\begin{array}{lr}
0 & \text { if } n \equiv 0 \text { or } 2(\bmod 3) \\
1 & \text { if } n \equiv 1(\bmod 3)
\end{array}\right.
$$

Proof: Obvious from Theorem 2.1.

The following Remarks are direct consequence of the Corollaries above. We have to note that if $G=P_{n}$, then $\varphi_{G}(n)=f_{G}(n)+g_{G}(n)+h_{G}(n)$.

Remark 2.2. Let $G=P_{n}$. If $n \equiv 0(\bmod 3)$, then $h_{G}(n)=g_{G}(n)<f_{G}(n)=\varphi_{G}(n)$.

Remark 2.3. Let $G=P_{n}$. If $n \geq 8$ and $n \equiv 1$ or $2(\bmod 3)$, then

$$
h_{G}(n)<g_{G}(n) \leq f_{G}(n)<\varphi_{G}(n) \text {. }
$$

The following results determine the probability of getting sets $D_{1}, D_{2}$, and $D_{3}$ in graph $G=P_{n}$ where $n \geq 1$ with certain restrictions.

Theorem 2.2. Let $G$ be a path of order $n \geq 3$. Then,

$$
P_{G}^{1}(n)= \begin{cases}1 & \text { if } n \equiv 0(\bmod 3) \\ \frac{n^{2}+n-2}{n^{2}+13 n+4} & \text { if } n \equiv 1(\bmod 3) \\ \frac{n-2}{n+4} & \text { if } n \equiv 2(\bmod 3)\end{cases}
$$

Proof: Let $G=P_{n}$ where $n \in \mathbb{N} \backslash\{1,2\}$. By getting the ratio of Corollary 2.1 and Theorem 2.1 , that is, $f_{G}(n) / \varphi_{G}(n)$, then this completes the proof.

Remark 2.4. Let $G=P_{n}$. If $n \leq 2$, then $P_{G}^{1}(n)=0$.

Theorem 2.3. Let $G$ be a path of order $n \geq 1$. Then,

$$
P_{G}^{2}(n)= \begin{cases}0 & \text { if } n \equiv 0(\bmod 3) \\ \frac{12 n-12}{n^{2}+13 n+4} & \text { if } n \equiv 1(\bmod 3) \\ \frac{6}{n+4} & \text { if } n \equiv 2(\bmod 3)\end{cases}
$$

Proof: Let $G=P_{n}$ where $n \in \mathbb{N}$. Then, by getting the ratio of Corollary 2.2 and Theorem 2.1, that is, $g_{G}(n) / \varphi_{G}(n)$. And this completes the proof.

Theorem 2.4. Let $G$ be a path of order $n \geq 4$. Then,

$$
P_{G}^{3}(n)=\left\{\begin{array}{lr}
0 & \text { if } n \equiv 0 \text { or } 2(\bmod 3) \\
\frac{18}{n^{2}+13 n+4} & \text { if } n \equiv 1(\bmod 3)
\end{array}\right.
$$

Proof: Let $G=P_{n}$ where $n \in \mathbb{N} \backslash\{1\}$. Hence, by getting the ratio of Corollary 2.3 and Theorem 2.1, that is, $h_{G}(n) / \varphi_{G}(n)$, then this completes the proof. 
Remark 2.5. Let $G=P_{n}$. If $n=1$, then $P_{G}^{3}(n)=0$.

By the definition of independent domination in graphs above, we obtained the following remark.

Remark 2.6. Let $G=P_{n}$. Then, the following holds:

i.) $\sum_{v \in D_{1}} \operatorname{deg}_{G}(v)=2 \gamma_{i}(G)$;

ii.) $\sum_{v \in D_{2}} \operatorname{deg}_{G}(v)=2 \gamma_{i}(G)-1$; and

iii.) $\sum_{v \in D_{3}} \operatorname{deg}_{G}(v)=2\left(\gamma_{i}(G)-1\right)$.

The following result determines the number of all possible ways of generating a dominating set in cycle graph $C_{n}$ where $n \geq 3$.

Theorem 2.5. Let $G=C_{n}$ where $n \in \mathbb{N} \backslash\{1,2\}$. Then,

$$
\left|S_{G}\right|=\varphi_{G}(n)=\left\{\begin{array}{cc}
6 & \text { if } n=4 \\
3 & \text { if } n \equiv 0(\bmod 3) \\
\frac{1}{18}\left(n^{3}-5 n^{2}+22 n\right) & \text { if } 7 \leq n \equiv 1(\bmod 3) \\
\frac{1}{3}\left(n^{2}-2 n\right) & \text { if } n \equiv 2(\bmod 3)
\end{array}\right.
$$

Proof. Suppose that $G=C_{n}$ where $n \in \mathbb{N} \backslash\{1,2\}$. Then, we consider that following 4 cases below:

Case 1. If $n=4$, then we have $|V(G) \backslash D|=|D|=2$. When the elements of $D$ are alternate with the elements of $V(G) \backslash D$, this implies that there are 2 possible arrangement of this form in graph $G$. On the other hand, if the elements of $D$ are connected in $G$, then there are 4 possible ways since the eccentricity of $u \in D$ to $v \in D$ is $e(u)=3$. Thus, $\left|S_{G}\right|=\varphi_{G}(n=4)=6$.

Case 2. Considering $n \equiv 0(\bmod 3)$, then $\forall u_{j} \in D, \exists a, b \in V(G) \backslash D$ such that $u_{j} \in N(a) \cap$ $N(b)$ and $\bigcap_{\text {all } j} N\left(u_{j}\right)=\varnothing$. Since $G$ is a cycle and $d_{G}\left(u_{j}, u_{j+1}\right)=3$, then this follows that $\left|S_{G}\right|=\varphi_{G}(n)=3$.

Case 3 . Let $7 \leq n \equiv 1(\bmod 3)$. Consider first a graph $H=P_{n}$. Then, we reflect on the following subcases below:

Subcase 1. By Remark 2.1 and Theorem 2.1, there is only one configuration of independent dominating set considering that there exists $u, v \in D=I$ such that $\operatorname{deg}(u)=\operatorname{deg}(v)=1$. Let $u_{1}, u_{2}, \ldots, u_{n}$ be the vertices of graph $H$. Then, $u_{1}$ and $u_{n}$ are dominating vertex. Now, to form a cycle graph we connect the two vertices $u_{1}$ and $u_{n}$ by adding an edge. This implies that $e\left(u_{1}\right)$ from $u_{n}$ is $n-1$ and it obviously follows that there are $n$ possible ways of generating dominating vertex of this form in graph $G$.

Subcase 2. Again, by Remark 2.1 and Theorem 2.1, there are $\frac{n^{2}-5 n+4}{18}$ arrangement of dominating set given that two vertices in $D$ are in the second vertex and second to last vertex of $H$. Let $v_{1}, v_{2}, \ldots, v_{n}$ be the vertices of path $H$. Connecting $v_{1}$ and $v_{n}$ by adding an edge, we form a cycle graph $G$. Since $e\left(v_{1}\right)=n-1$ from vertex $v_{n}$, then this implies that there are $n\left(\frac{n^{2}-5 n+4}{18}\right)$ ways to generate dominating vertices of this form. If we combine the two sub 
cases, then we obtain, $\left|S_{G}\right|=\varphi_{G}(n)=n\left(\frac{n^{2}-5 n+4}{18}\right)+n$. Simplifying the equation, we end up with,

$$
\left|S_{G}\right|=\varphi_{G}(n)=\frac{n^{3}-5 n^{2}+22 n}{18} .
$$

Case 4. Lastly, we let $n \equiv 2(\bmod 3)$ and $H=P_{n}$. Then, by Remark 2.1 and Theorem 2.1 , there are $\frac{n-2}{3}$ configuration in graph $H$ considering that $\forall u_{j} \in D, \operatorname{deg}\left(u_{j}\right)=2$. Let $u_{1}, u_{2}, \ldots, u_{n}$ be the vertices of graph $H$. By connecting $u_{1}$ and $u_{n}$ by adding an edge, we obtain a cycle graph $G$. Since $e\left(u_{1}\right)=n-1$ from vertex $u_{n}$, then it follows that $\left|S_{G}\right|=\varphi_{G}(n)=n\left(\frac{n-2}{3}\right)=\frac{n^{2}-2 n}{3}$. Hence, combining all cases and this completes the proof.

By the Theorem 2.5 above, we obtain the following remarks.

Remark 2.7. Let $G=C_{n}$ where $n \geq 3$. Then, $\varphi_{G}(n)=f_{G}(n)$ and $g_{G}(n)=h_{G}(n)=0$.

Remark 2.8. Let $G=C_{n}$ where $n \geq 3$. Then, $P_{G}^{1}(n)=1$ and $P_{G}^{2}(n)=P_{G}^{3}(n)=0$

\section{CONCLUSION}

This paper constructed a new combinatorial formulae that counts the number of ways of putting dominating set in path and cycle graphs. These formulae are function of the order of the said two graphs.

\section{REFERENCES}

[1] Allan, R. B. \& Laskar, R., "On domination and independent domination number of a graph,” Discrete Mathematics, vol. 23, pp. 73-76, 1978.

[2] Arocha, J. L. \& Llano, B., "The number of dominating k-sets of paths, cycles and wheels", arXiv preprint:1601.01268, 2018.

[3] Casinillo, L. F., "A note on Fibonacci and Lucas number of domination in path", Electronic Journal of Graph Theory and Applications, vol. 6, no. 2, pp. 317-325, 2018.

[4] Casinillo, L. F., "Odd and even repetition sequences of independent domination number", Notes on Number Theory and Discrete Mathematics, vol. 26, no. 1, pp. 8-20, 2020.

[5] Casinillo, L. F., Lagumbay, E. T. \& Abad, H. R. F., "A note on connected interior domination in join and corona of two graphs, " IOSR Journal of Mathematics, vol. 13, no. 2, pp. 66-69, 2017.

[6] Chartrand, G. and Zhang, P., A First Course in Graph Theory, New York: Dover Publication, Inc., 2012.

[7] Cockayne, E. J., \& Hedetniemi, S. T., "Towards a theory of domination in graph," Networks Advanced Topics, vol. 7, pp. 247-261, 1977.

[8] Goddard, W. \& Henning, M. A., "Independent domination in graph: A survey and recent results," Discrete Mathematics, vol. 313, pp. 839-854, 2013.

[9] Haynes, T. W., Hedetniemi, S. T., \& Slater, P. J., Fundamentals of Domination in Graphs, New York: Marcel Dekker, 1998.

[10] Ore, O., Theory of Graphs, American Mathematical Society Provedence, R. I., 1962.

[11] Perderson, A. S., \& Vestergaard, P. D., "The number of independent sets in unicyclic graphs," Discrete Applied Mathematics, vol. 152, pp. 246-256, 2005. 
[12] Tarr, J. M., Domination in Graphs. Graduate Theses and Dissertations. Retrieved from https://scholarcommons.usf.edu/etd/1786, 2010.

[13] Samanmoo, R., Trakultraipruk, N., \& Ananchuen, N., " $\gamma$-Independent dominating graphs of paths and cycles," Maejo International Journal of Science and Technology, vol. 13, no. 3, pp. 245-256, 2019. 